\title{
Neue Strategie gegen einen alten Gegner
}

— Eine latente Tuberkuloseerkrankung kann zum Problem werden. Denn was da symptomlos schlummert, ist nicht leicht zu diagnostizieren und kann immungeschwächte Patienten ernsthaft gefährden. Mit einer neuen Klasse von Immuntests, den Interferon-Gamma-Release-Assays (IGRA), ist das Screening von Risikopatienten einfacher und treffsicherer möglich als mit den bisherigen Hauttests.

Von einer latenten Tbc spricht man, wenn sich das Mycobacterium tuberculosis im Körper befindet, aber noch keine klinisch nachweisbare Erkrankung vorliegt. Doch eine solche latente Tbc kann jederzeit zu einer manifesten Erkrankung führen. „Besonders gefährdet sind Patienten mit Autoimmunkrankheiten wie rheumatoide Arthritis oder M. Crohn, wenn eine Therapie mit einem Tumor-Nekrose-Faktor-(TNF-)alpha-Blocker eingeleitet wird“, sagte PD Dr. Roland Diel, Hamburg. Diese Patienten hätten ein vielfach erhöhtes Erkrankungsrisiko. Auch sei die Diagnostik einer manifesten Tbc bei diesen Patienten nicht immer einfach, da sie in ca. 50\% der Fälle extrapulmonal sei. „Das Problem ist, dass bei diesen Patienten eine latente Tbc mit dem Tuberkulinhauttest nicht ausgeschlossen werden kann“, so Diel.

Eine große Bereicherung der Tbc-Diagnostik sind daher die neuen IGRA. Mit diesem innovativen Bluttest wird die zellvermittelte Immunreaktion mit Tbc infizierter Patienten nachgewiesen. Dabei werden dem Vollblut hoch spezifische Antigene zugeführt, was bei Tbc zur Produktion des Zytokins Interferon-(IFN-)Gamma führt., ,Falls die IFN-Gamma-Konzentration im Tbc-Antigenröhrchen den Grenzwert übersteigt, liegt bei dem Getesteten mit hoher Wahrscheinlichkeit eine Tbc-Infektion vor", so Diel. Dies sei der erste große wissenschaftliche Fortschritt gegenüber dem bisher verwendeten über 100 Jahre alten Hauttest.

Mit QuantiFERON ${ }^{\circledR}$ TB Gold In-Tube (OFTTM) steht ein solcher Test zur Verfügung, der eine höhere Präzision und Zuverlässigkeit beim Tbc-Screening bietet als bisher. In Studien waren Sensitivität und Spezifität signifikant höher als beim Hauttest. „Die Sensitivität von OFTTM liegt bei fast 90\%, die Spezifität bei fast 100\%", sagte Diel.

Für die Anwendung eines IGRA spricht auch, dass nicht zwei Arztbesuche nötig sind. Der Test wird auch nicht durch eine BCG-Impfung verfälscht, und nicht tuberkulöse mykobakterielle Infektionen führen nicht zu einem falsch-positiven Ergebnis. Das Ergebnis unterliegt zudem nicht wie beim Hauttest individuellen Interpretationsschwankungen des Arztes.

Kosten für etwaige Zusatzuntersuchungen, v. a. Röntgen-Thorax, werden gesenkt.,,Aufgrund der hohen Zuverlässigkeit ist ein 2Schritt-Test, d.h. erst Tuberkulinhauttest, dann evtl. IGRA, heute nicht mehr sinnvoll“, so Diel. Der Nachweis einer latenten Tbc erfordere bei Patienten mit einer Indikation für eine TNF-alpha-Blocker immer eine Chemoprävention, da bei jedem Fünften mit dem Blocker mit einer manifesten Tbc gerechnet werden müsse.

\section{ps}

Satellitensymposium beim 51. Pneumologenkongresses, 18.3.2010 in Hannover, Veranstalter: Cellestis

\section{Gegen Emphysemprogression bei Alpha-1-Antitrypsin-Mangel}

\section{Dauersubstitution empfohlen}

- Alpha-1-Antitrypsin-( $\alpha 1-A T-)$ Mangel beruht auf einer genetischen Störung, charakterisiert durch eine verminderte $\alpha 1$-ATKonzentration im Serum. Betroffene haben ein erhöhtes Risiko, Lungenerkrankungen bis hin zum Emphysem zu entwickeln. Beim Pneumologenkongress in Hannover wurde interdisziplinär über die Vorteile einer $\alpha 1-$ AT-Substitution diskutiert.

$\alpha 1$-AT ist ein vorwiegend in der Leber gebildeter Proteinaseinhibitor und wird im Blut und im Oberflächenfilm des Atemwegsepithels gefunden. Es ist die häufigste Antiproteinase im Blutkreislauf des Menschen. Sie bietet einen über 9o-prozentigen Schutz gegen die neutrophile Elastase im tiefen Respirationstrakt [1]. Neutrophile Elastase wirkt bei der sekundären Immunabwehr gegen Infektionen oder Entzündungen. Sie kann aber gesundes Lungengewebe schädigen, wenn sie nicht durch $\alpha 1$-AT neutralisiert wird. Der a1-AT-Mangel gehört zu den häufig erst spät oder gar nicht erkannten Ursachen von Atemwegserkrankungen. Besondere jüngere ( $<50$ Jahre) und nicht rauchende COPD-Patienten sollten laut der Empfehlung der deutschen COPD-Leitlinie von 2007 einmal im Leben im Labor ihren $\alpha 1-A T-S p i e g e l$ testen lassen. Der differenzialdiagnostische Verdacht auf einen Mangel muss durch die quantitative Bestimmung des $\alpha_{1}$-AT-Spiegels im Serum bestätigt oder widerlegt werden.

\section{Humanes AT-Präparat schützt}

Wie Prof. Karl Wegscheider, Hamburg, Experte für medizinische Biometrie und Epidemiologie, sagte, konnte in klinischen Studien gezeigt werden, dass eine Substitutionstherapie mit einem humanen $\alpha 1$ AT (Prolastin ${ }^{\circledR}$ ) schützende $\alpha 1$-AT-Spiegel aufrecht erhalten kenn. Die Progression des Lungenfunktionsverlusts (reduzierte $\mathrm{FEV}_{1}$ ) wird verlangsamt und die Mortali- tät sinkt signifikant. Die Ergebnisse zu humanem $\alpha 1$-AT wurden allerdings nur in offenen, nicht randomisierten Studien gewonnen.

Aber, so erläuterte der Pneumologe Dr. Thomas Köhnlein, Hannover: „Die Effekte der pulmonalen pharmakologischen Interventionen, inklusive der Substitutionstherapie bei $\alpha 1-A T-M a n g e l$, sind mit den herkömmlichen Endpunkten für klinische Studien nicht suffizient darstellbar.".

Wie der Vorsitzende, Prof. Roland Buhl, Mainz, in seinem Fazit sagte, wird es eine randomisierte Studie zu humanem $\alpha 1-A T$ mit dem Endpunkt FEV 1 wohl nie geben. Die weitere Entwicklung wird durch fortdauernde klinische Beobachtung dieser Substitutionstherapie aber nach seiner Meinung „spannend bleiben“. js

1. Crystal RG. Alpha-1-antitrypsin deficiency: pathogenesis and treatment. Hosp Pract 1991:81-94.

Symposium beim 51. Pneumologenkongress, 17. 3.2010 in Hannover. Veranstalter: Talecris Biotherapeutics $\mathrm{GmbH}$. 